\section{GESTIÓN EFICIENTE DE LA EVALUACIÓN CONTINUA DEL ALUMNADO. LA INTEGRACIÓN DEL TRABAJO DE ESCRITORIO CON MOODLE}

\author{
E. Querol \\ Dpto. de Ingeniería Química y Combustibles, \\ Universidad Politécnica de Madrid \\ E.T.S.I. Minas, Alenza 4, 28003 Madrid \\ enrique.querol@upm.es
}

J. L. Perez-Benedito

Dpto. de Vehículos Aeroespaciales, Universidad Politécnica de Madrid E.U.I.T. Aeronáutica, Plaza del Cardenal Cisneros 3, 28040 Madrid joseluis.perez@upm.es

\author{
M. J. García-Martínez \\ P. Segarra Catasús \\ Dpto. de Ingeniería Química y Combustibles, \\ Universidad Politécnica de Madrid. \\ E.T.S.I. Minas, Alenza 4, 28003 Madrid \\ mj.garcia@upm.es,pablo.segarra@upm.es
}

ABSTRACT: A Visual Basic application for Microsoft Excel 2010 has been developed and presented. The aim is to provide a tool through Moodle platform that helps professors to manage exams, works and tasks in courses with continuous assessment. Its application in a specific subject is also presented.

KEY WORDS: Moodle; VBA; Excel; continuous evaluation.

\section{INTRODUCCIÓN}

Se ha desarrollado una aplicación (en adelante GEECA) programada en VBA (Visual Basic for Applications) en el entorno Microsoft Excel 2010 como herramienta necesaria para aliviar el trabajo del profesorado en la recuperación de la información de las pruebas de evaluación continua realizadas a los estudiantes. GEECA está diseñada para interactuar con Moodle (1.9.12), que es el Ambiente Educativo Virtual (AEV) o sistema de administración de cursos utilizado en la Universidad Politécnica de Madrid.

\section{EFFICIENT MANAGEMENT OF THE CONTINUOUS EVALUATION SYSTEMS. INTEGRATION OF THE PERSONAL DESKTOP WITH MOODLE}

RESUMEN: Se presenta una aplicación desarrollada en Visual Basic para Microsoft Excel 2010 con el propósito de ofrecer una herramienta que ayude al profesorado en las labores de calificación de pruebas de evaluación continua a lo largo de un curso en conjunción con la plataforma Moodle. Se describe también su aplicación a una asignatura concreta.

PALABRAS CLAVE: Moodle; VBA; Excel; evaluación continua.

En la actualidad existe bastante rechazo por parte de los profesores a entrar en la dinámica del uso de Moodle debido a que es un entorno que se desconoce, y que tiende a utilizarse meramente como un medio de intercambio de contenidos (Lonn y Teasley, 2009). El aprendizaje es algo más que eso, y debe potenciarse el uso del $A E V$ para permitir la nivelación de estudiantes, motivar al estudiante, y provocar el intercambio social entre los alumnos según Lonn y Teasley (2009).

Las tareas que se realizan con menos frecuencia provocan en el usuario rechazo y poca predisposición. Estos son al- 
gunos ejemplos: Actualización de fechas de las actividades Moodle al inicio del nuevo curso académico; Incorporación de nuevos cuestionarios; Actualización de las preguntas y actividades de evaluación; Generación de grupos. Asimismo el rechazo es grande ante actividades frecuentes, pero muy rutinarias o tediosas: Recopilación de notas de distintos profesores y análisis de las incidencias; Recopilación de archivos subidos por alumnos para cumplir una tarea; Incorporación a Moodle de las notas de una actividad offline; Correcciones.

Aunque la tendencia a seguir es que las aplicaciones favorezcan la flexibilidad de trabajo, el camino se prevé todavía muy largo, por lo que tenemos necesidad de ciertas herramientas que nos ayuden con tareas simples, repetitivas y programables. Este ahorro de tiempo facilita la comunicación entre nuestro día a día y el uso momentáneo del $A E V$ en tareas realizadas ocasionalmente. GEECA pretende simplificar el proceso de adaptación a Moodle, mientras el $A E V$ se perfecciona.

Los objetivos perseguidos son la disminución del tiempo empleado por el profesor en las tareas de recolección de las tareas solicitadas a los alumnos así como facilitar el proceso de corrección, permitiendo una corrección más automatizada, rápida y con criterio de calificación más uniforme. Esto evita las pérdidas del ritmo de aprendizaje según Yengin, Karahoca y Yücel (2008) resultantes de largos tiempos de corrección que desembocan en el desinterés del alumno y en la confusión de conceptos al coincidir en el tiempo las correcciones de unos temas con las clases de temas posteriores. Se pretende con ello lograr una herramienta de amplia aceptación, al estilo de la conocida macro en Microsoft Word de cuestionarios como en Clemente (2011), que permite la vinculación directa de la herramienta institucional con el ámbito de trabajo del profesor.

Se propone también, como ejemplo, la aplicación de unos criterios de corrección rápidos y precisos a una asignatura. El objeto es hacer más evidente cómo obtener el mejor provecho posible a la conjunción de la herramienta con Moodle y con la guía de aprendizaje de la asignatura. Por último, se describe la aplicación de GEECA a una asignatura concreta así como una descripción de las virtudes y defectos. Se dan también orientaciones sobre cómo estructurar las guias de aprendizaje, asi como las posibilidades de aplicación en el entorno educativo de grado y postgrado.

\section{UNA APLICACIÓN ORIENTADA A LA GESTIÓN EFICIENTE DE LA EVALUACIÓN}

Por parte del profesor, el tiempo dedicado a corregir y a tareas de coordinación es inmenso o al menos así lo parece. La decepción se muestra en seguida en los rostros de quienes han decidido dedicar una semana a corregir, con el objetivo de disminuir, el montón de "pendientes de corregir". Por parte del alumno es imprescindible para su motivación positiva ir viendo metas, logros, y que su trabajo se vea valorado. La motivación del estudiante es imprescindible para una educación de calidad según Kotsiantis, Patriarcheas y Xenos (2010).

Las asignaturas presenciales con Moodle inicialmente se benefician de la presencia electrónica de materiales. Ello beneficia al estudiante al poder pasar su documentación al e-Book/Netbook/Tablet correspondiente con una excelente calidad y visualización, así como para evitar las colas y costes asociados a los servicios de reprografía, a la par que se disminuye el peso transportado. Los tiempos cambian, el lenguaje también y hay que hacer un esfuerzo por adaptarse a la mentalidad y formas de comunicarse del estudiante, en la misma medida que el estudiante debe adaptarse a la asignatura. Los estudiantes tienen gran tendencia a usar $T I$, bastante mayor que los profesores según Lonn y Teasley (2009). El uso de Moodle permite hablar el mismo idioma, pero como el profesor es minoria debe hacer uso de tres armas simultáneamente: su conocimiento especializado, la planificación de la asignatura antes de su inicio, y el uso de herramientas informáticas y docentes a su alcance. En un paso posterior es posible que el profesor aproveche algunas de las múltiples posibilidades de Moodle y cambie con ello la forma de dar clase como en Lonn y Teasley (2009).

\subsection{La rúbrica de corrección estricta}

Existen diversos métodos para simplificar las labores de corrección de evaluación continua. El método recomendado para simplificar la corrección es corregir cada apartado como bien o mal. Para ello se sugiere que sólo se den por buenos los resultados de los apartados que son correctos y que cuentan con las operaciones adecuadas para llegar a ellos. La utilización de hojas con espacios para las contestaciones en limpio y espacios para los planteamientos y operaciones son sin duda de gran ayuda para poder corregir cada apartado de un vistazo. 
Esta forma de corregir disminuye mucho el tiempo de corrección y en caso de utilizar Moodle o los Clickers la corrección puede ser automática, con una revisión rápida por parte del profesor. Es imprescindible, adicionalmente, que el alumno pueda revisar rápidamente la resolución correcta del ejercicio para aprovechar el interés natural de contrastar su ejercicio con el resuelto y comprobar así si su nota es correcta y por qué. Este momento es especialmente importante ya que es un momento de gran intensidad que hay que aprovechar: el momento de interés y necesidad. La motivación afecta directamente a la forma en que las personas aprenden según Henderlong, McClintic y Hayenga (2009).

\subsection{Tareas de subida de archivos.}

Esta tarea (figura 1 izda) permite que el alumno resuelva ejercicios más elaborados en el entorno de un libro de cálculo o similares, asociando varias partes de la asignatura o conceptos de varias materias para llegar a la resolución del ejercicio. La propuesta de resolver dichas tareas, en un libro de cálculo de Excel, tiene la ventaja de forzar al alumno de ingeniería a utilizar una herramienta ofimática muy extendida, y que se le pedirá sin duda en su entorno profesional.

Adicionalmente, para el profesor sólo es necesario marcar unas celdas en las que el alumno realice la última opera- ción que da el resultado final del apartado en las unidades pedidas, pero con la libertad de poder resolver el ejercicio según su criterio. Si el alumno lo desea puede incluso mover las celdas de resultado a la ubicación más conforme con su resolución, para lo cual sólo hace falta añadir una hoja adicional vinculada a los valores de las celdas.

Por lo tanto, existen dos formas de asegurar que GEECA será capaz de recopilar los datos realizados por el alumno. Por un lado, conviene siempre forzar al alumno a que resuelva el ejercicio en una única hoja del libro de cálculo. Si se dejó el hueco adecuado para la resolución de cada apartado la celda para el resultado final puede tener una posición fija, a la cual apunte GEECA en la recogida de datos. Otra opción es situar las celdas para cada apartado en un sitio común, de manera que el alumno mueva dichas celdas hasta el lugar que le resulte conveniente para ubicar la resolución del apartado. En este último caso es donde se requiere que el libro enunciado disponga de una hoja para el enunciado y la resolución por parte del alumno, y otra de recopilación de los datos de las celdas.

GEECA lee la plantilla de recopilación de datos que previamente ha realizado el profesor (figura 1 dcha), y que está situada en una hoja cuyo nombre empieza por "MT_" (iniciales elegidas por Moodle Task). Dicha hoja indica qué

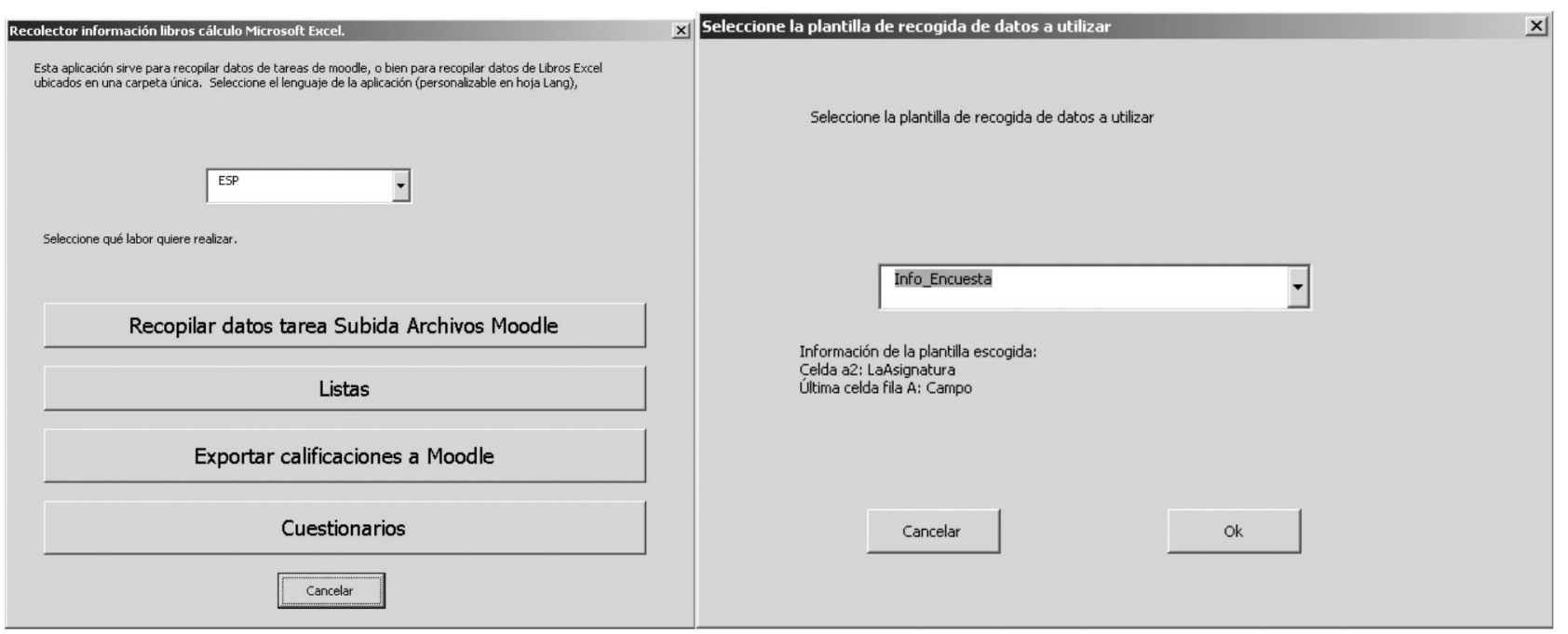

Figura 1. Pantalla inicial de GEECA, recopilación de datos (izda) y selección de plantilla (dcha). 
hoja hay que leer y qué celda dentro de dicha hoja. Al indicar ambos valores GEECA puede leer datos de más de una hoja del libro de cálculo.

La elaboración de los ejercicios requiere por tanto un tiempo adicional de planificación y preparación que se ve muy recompensado por la facilidad de recogida de datos y de calificación. El beneficio es tanto mayor cuanto mayor sea el grupo, o cuantos más cursos académicos empleen las mismas plantillas (pidiendo la resolución de apartados equivalentes, pero cambiando los enunciados). Así, aunque la preparación de enunciado y plantilla es ligeramente laboriosa, sólo hay que realizarlo una vez, y permite cambiar el enunciado de la tarea de bloque, que si está bien elegido será igual todos los años (aunque con enunciado variado, para evitar que algún alumno haga labores de copia, en lugar de labores de estudio).

GEECA guía al usuario mientras le solicita los datos necesarios, que desembocan en que el usuario comprima las carpetas Moodle con los datos (cada carpeta tiene como nombre el Moodle ID de cada alumno y en su interior contiene todos los archivos que haya subido el alumno) y lo descargue al ordenador.

GEECA entonces comienza descomprimiendo los archivos y copiándolos a la carpeta base, renombrándolos con el Moodle ID de cada alumno (para evitar repeticiones) seguido de un número para identificar cada uno de los archivos subidos por el alumno. Acto seguido el profesor tiene la posibilidad de indicar cómo quiere renombrar los archivos, para lo cual se hace uso de un libro de calificaciones de la asignatura descargado de Moodle.

Esto de por sí es ya un ahorro de tiempo importante, puesto que para evaluar estas tareas el profesor tenía que ir descargando y abriendo los archivos uno a uno, y en caso de guardarlos en el ordenador ir asignándoles a mano un nombre. Posteriormente, GEECA solicita al profesor que seleccione la plantilla de recopilación de datos que desee usar y genera a partir de ello un libro en el que se recopilan los datos de todos los libros subidos por todos los alumnos en una única tabla.

Esta tabla facilita la calificación, ya que se puede ir mirando columna por columna corrigiendo simultáneamente y de manera rápida los resultados, teniendo asimismo la posibilidad de abrir rápidamente el libro original del estudiante para comprobar las operaciones realizadas.

\subsection{Cuestionarios y exámenes}

GEECA también facilita la elaboración de cuestionarios, permitiendo al profesor seleccionar la batería de preguntas a utilizar, y elegir cuántas preguntas y variantes de cuestionario quiere realizar, así como ver las preguntas y respuestas del cuestionario según se va formando. Tras dicha elección se le pregunta al profesor qué preguntas quiere incluir y si quiere generar un archivo en formato XML Moodle para su importación desde Moodle. De esa manera, el profesor puede recopilar sus preguntas en Excel, e incluir la retroalimentación (Feedback) de las mismas si son operaciones. En todo caso GEECA genera las variantes deseadas barajando las preguntas y las respuestas, y ofreciendo una tabla de corrección rápida.

\subsection{Coordinación de listas}

En grupos grandes es habitual que existan múltiples profesores, por lo que se hace imprescindible una tarea de coordinación y de control. La herramienta usual para estos trabajos es Excel. Habitualmente el coordinador dispone de una lista maestra y recopila manualmente los datos que ofrece cada profesor. GEECA permite recopilar fácilmente los datos de las listas auxiliares en la lista maestra, e incluso permite utilizar una columna identificativa de grupo para poder marcar las ausencias de alumnos o bien la asistencia de un alumno de otro grupo.

A las largas listas de alumnos y la gran cantidad de singularidades que acontecen en el día a día de los alumnos que rompen la agenda del profesor, se une la compleja interacción con gran número de profesores: manejo de listas diferentes, entrega de notas en tiempos distintos, puntos de vista distintos en la gestión de la información, dificultad para el uso del Moodle... lo cual no hace sino aumentar el tiempo dedicado a estas tareas.

\subsection{Exportación de notas en Excel para subir a Moodle}

GEECA permite recopilar de un listado Excel los DNI (ó email) y las notas, generando un archivo CSV que se puede 
importar en Moodle. Para ello se le pide al profesor que identifique el libro de cálculo, el primer valor de DNI y de nota a exportar. Acto seguido se escribe el archivo CSV en formato UTF-8 para su importación en Moodle. De esta manera el profesor puede mantener su libro de calificaciones en Excel en su ordenador, de manera independiente a las calificaciones de Moodle, y subir a éste las calificaciones en el momento deseado.

\section{Aplicación a una asignatura}

Una asignatura de menos alumnos, pero muy interactiva orientada a proyectos, también puede beneficiarse de GEECA. Es el caso de la asignatura Termoeconomía y Optimización Energética (Querol, García-Torrent, Cámara y Montes, 2011). Como puede verse en la guía de aprendizaje, la planificación temporal de la asignatura conlleva el avance en la teoría del libro (Montes, García y Querol, 2009) seguido de su aplicación inmediata a una instalación que es objeto del análisis paulatino por parte de toda la clase. Adicionalmente, se realiza en grupos de cuatro alumnos el análisis de una instalación en paralelo.

Esta interactividad conlleva, en la asignatura de cuatro horas a la semana, la corrección de habitualmente una prueba simple en papel y dos tareas subidas a Moodle cada semana. Tal cantidad de trabajo con el alumno sólo es posible en grupos de últimos cursos con menor número de alumnos que permiten hacer una asignatura muy práctica y menos teórica.

Hasta el momento GEECA se ha aplicado para: la realización de cuestionarios en clase, con un importante ahorro de tiempo en la elaboración de los mismos; la recopilación de la información de las horas de dedicación de cada alumno a la semana, y también para el seguimiento del trabajo de los grupos (mediante encuestas) y su satisfacción; Por último se ha empleado para subir los resultados de las pruebas de evaluación offline, de ejercicios rápidos realizados en aula de teoría.
En el curso 2011/2012 se adaptarán los enunciados de los libros Excel para recopilar también los resultados de las dos tareas que realizan los alumnos en Excel y que suben a Moodle antes de una fecha y hora límite. Hasta el momento la corrección se realizaba manualmente, evaluando a cada alumno descargando su libro, cambiando el nombre del mismo, abriéndolo, corrigiéndolo y subiendo a Moodle la nota y el comentario a la corrección. El trabajo inicial para aprovechar las funcionalidades de GEECA es grande, pero al ser un trabajo bastante sencillo se puede distribuir el trabajo entre los profesores de la asignatura trabajando en paralelo o emplear tiempos sueltos. La inversión en el tiempo inicial se recupera rápidamente durante el curso ya que aunque cada año hay que adaptar los enunciados a la instalación que se analiza, los apartados son siempre los mismos por lo que cada año habrá que cambiar exclusivamente los encabezados de los enunciados.

\section{Conclusión}

GEECA mejora la experiencia de corrección del profesorado, facilitando la realización de correcciones rápidas así como la uniformidad de criterio al poder evaluar apartado tras apartado. La integración del uso de Moodle, GEECA y las pruebas de evaluación continua constituye una sinergia que permite mejorar la calidad del tiempo del profesor, detectar los fallos comunes de manera más sencilla, y ahorrar en el proceso de evaluación un tiempo puramente administrativo que puede emplearse para la mejora de los aspectos docentes de la asignatura.

GEECA es multilingüe. Los idiomas actualmente incorporados son Español e Inglés, pero basta introducir una columna adicional a la hoja Lang del libro para incorporar un nuevo idioma. GEECA está disponible gratuitamente para quien la solicite por correo electrónico a los autores con el único requisito de citar el presente artículo en caso de su utilización para una publicación. Es intención de los autores ir adaptando GEECA a las versiones de Moodle que se vayan instalando en la UPM. 


\section{BIBLIOGRAFÍA}

Clemente Correa Cervantes, A. (2011): PlantiIla para generarcuestionarios en formato GIFT en español. http://moodle.org/mod/ forum/discuss.php?d=46432\#212950. Consulta: 2011-05-25.

Henderlong Corpus, J.; Mcclintic-Gilbert, M. S. y Hayenga, A. O. (2009): "Withinyear changes in children's intrinsic and extrinsic motivational orientations: Contextual predictors and academic outcomes", Contemporary Educational Psychology, vol. 34, pp. 154-166.

Kotsiantis, S.; Patriarcheas, K. y Xenos, M. (2010): "A combinational incremental ensemble of classifiers as a technique for predicting students' performance in distance education", Knowledge-Based Systems, vol. 23-6, pp. 529-535.
Lonn, S., Teasley, S. D. (2009): "Saving time or innovating practice: Investigating perceptions and uses of Learning Management systems", Computers \&t Education, vol. 53, pp. 686-694.

Montes Villalón, J.; García Torrent, J. y Querol, E. (2009): Termoeconomía y optimización energética, Fundación Gómez Pardo, ISBN: 978-84-6928320-2, Madrid.

Querol, E.; García-Torrent, J.; Cámara, A. y Montes, J. M. (2011): Termoeconomía y optimización energética. http://ocw. upm.es/explotacion-de-minas

Yengin, I.; Karahoca, D.; Karahoca, A. y Yücel, A. (2010): "Roles of teachers in e-learning: How to engage students \&t how to get free e-learning and the future", Procedia Social and Behavioral Sciences, vol. 2, pp. 5775-5787. 